\title{
The homogeneity effect in counting
}

\author{
ROBERT W. FRICK \\ University of Washington, Seattle, Washington
}

\begin{abstract}
In Experiment 1, college students counted digits presented in one row. In the homogeneous condition, the digits were identical to one another; in the heterogeneous condition the digits were different. Homogeneous rows of digits were counted more slowly and less accurately. In Experiments 2 and 3, this "homogeneity" effect remained when a space was inserted between each digit, when a heterogeneous border was put around the digits, and when subjects used a finger to count each digit one by one. However, the homogeneity effect was eliminated when the digits were presented on three different levels. Presenting the digits on three levels also improved counting in the heterogeneous condition. These results are consistent with the hypothesis that the Gestalt principles of grouping can act preattentively against the process of isolating each object to be counted.
\end{abstract}

The standard model for counting a set of objects seems to be to locate an object, say "one" (out loud or subvocally), locate a second object, say "two," and so on, pairing each object with a number from the sequence "one, two, three, ...." The number paired with the lastcounted object corresponds to the total number of objects. There are several components to this process of counting. One component is being able to recite the sequence "one, two, three, ...." A second component is ordering the objects linearly, such that each object is counted exactly once. For example, a line of children is easier to count than a playground full of children. A third component is that the objects have to be isolated and identified as individual units. For example, the number of drops in a glass of water cannot be counted without some operation to separate the drops from one another.

In Experiment 1, college students were asked to count a row of digits as quickly and accurately as possible. The issue was whether counting would be more difficult when the digits were all the same (e.g., 16 sevens) or when a mixture of different digits was presented. The former will be called the homogeneous condition and the latter the heterogeneous condition. College students, of course, know how to recite the sequence "one, two, three, ...,", and the digits were presented in a single row so there was no difficulty in ordering the digits. Finally, the digits were not adjoining and seemed to be easy to isolate. Thus, there was no obvious difficulty in counting the digits. Furthermore, given the model presented above for counting, there was no obvious reason why a homogeneous row of digits would be more difficult to count than a heterogeneous row of digits.

Beckwith and Restle (1966) suggested that a homogeneous row of objects should be no more difficult to count

\footnotetext{
I thank Ann Hutchinson, Meilene Chang, and Rosalba Mayorga for testing subjects and Darla Broberg, Lester Krueger, and an anonymous reviewer for commenting upon earlier drafts of this manuscript. Reprint requests should be sent to Robert Frick, Department of Psychology NI-25, University of Washington, Seattle, WA 98195.
}

than a heterogeneous row of objects. Notwithstanding this prediction, in their experiment, homogeneous rows of objects were counted more slowly. They noted this result but presented no statistical verification. Similarly, in a simpler task than counting, one in which objects were presented in two rows and children were asked to point to each object exactly once, homogeneous rows of objects were less accurately pointed to than heterogeneous rows of objects (Potter \& Levy, 1968). Again, no statistical verification was presented. Thus, despite no obvious reason for why a homogeneous row of objects might be more difficult to count, two experiments have reported this finding with no statistical verification. Experiment 1 investigated whether a homogeneous row of objects is actually more difficult to count.

\section{EXPERIMENT 1}

\section{Method}

Subjects. Thirty students from introductory psychology classes at the University of Washington volunteered in order to earn extra credit toward their course grades.

Design. There were two conditions, a homogeneous condition and a heterogeneous condition. There were 20 trials for each condition, and each number of digits was presented once. The order of these trials was randomized. Preceding these $\mathbf{4 0}$ trials were 5 practice trials, selected randomly from the set of possible trials.

Stimuli. On each trial, from 1 to 20 digits were presented simultaneously in one horizontal row. The digits 1 to 9 were used. The first (leftmost) digit of each presentation was selected randomly. In the homogeneous condition, the remaining digits were the same as the first digit. In the heterogeneous condition, the remaining digits were selected randomly with the constraint that no two adjacent digits could be identical.

The digits were presented on an NEC monitor, Model JB-1201M, controlled by an Apple II+ computer. Each digit was approximately $5 \mathrm{~mm}$ high and $3.5 \mathrm{~mm}$ wide. The digits were separated by $1 \mathrm{~mm}$ and were viewed from a distance of approximately $.75 \mathrm{~m}$. The first digit was always positioned in the same location.

Procedure. The subjects were tested individually. The procedure for the experiment was explained to each subject before the experiment was begun. The subject was instructed to count both quickly 
and accurately, although he or she was also told not to restart counting, but to continue counting even if unsure about whether he or she had counted the digits correctly. Finally, the subject was instructed to count the digits one by one.

Each trial began with the message "READY" on the computer screen. When the subject responded by saying "ready," the experimenter pressed a computer key. A dash then appeared and disappeared twice in the location that the first digit was to be presented. The dash and the digits were presented at an even tempo, such that the dash also cued when the digits would be presented. The digits were then presented and remained on the computer screen until the subject responded. The subject counted these digits silently and spoke the answer.

As soon as the subject spoke the answer, the experimenter pressed the space bar on the computer. The computer measured the time between the presentation of the digits and the keypress. This method of measuring response latency was unsophisticated, but it was unbiased and sufficiently reliable for this experiment. The experimenter then entered the subject's answer into the computer, ending the trial. When there was an irregularity in the trial, which happened infrequently, the experimenter voided the trial. In that case, the results of the trial were not recorded and another trial with the same condition and number of digits became the last trial. Testing for each subject was completed in 15 to $20 \mathrm{~min}$.

\section{Results}

After testing, one subject reported estimating the number of digits rather than counting them, and another subject reported sometimes counting only half the digits and then doubling the answer. The data from these two subjects were discarded from the following analyses (although the direction of their results tended to be consistent with the to-be-reported findings).

Two obvious dependent measures are the overall response latency and accuracy. A third useful dependent measure is the estimated time required to count each digit. This third measure was calculated as follows: An individual subject's performance can be graphed in a manner similar to that shown in Figure 1, with the number of digits to be counted placed on the abscissa and the response latency placed on the ordinate. In this graph, the slope of the line represents the estimated time to count one digit. Figure 1 presents the averages across all subjects. As shown in the figure, and as found in previous experiments (e.g., Chi \& Klahr, 1975), the response latency does not begin to increase very much until there are about five digits in the presentation. Therefore, a slope for each subject and for each condition was calculated considering only trials on which five or more digits had been presented.

Descriptive statistics for each condition are presented in Table 1. The results were the same for all three dependent measures: The subjects were slower overall to respond $[t(27)=5.92, p<.001]$, slower per digit $[t(27)$ $=3.77, p<.001]$, and less accurate $[t(27)=3.65$, $p<.01]$ in the homogeneous condition than in the heterogeneous condition. The robustness of the homogeneity effect is suggested by the number of subjects who showed the effect. As shown in Table 1, almost all of the subjects responded more quickly in the heterogeneous condition, and most of the subjects were more accurate in the heterogeneous condition.

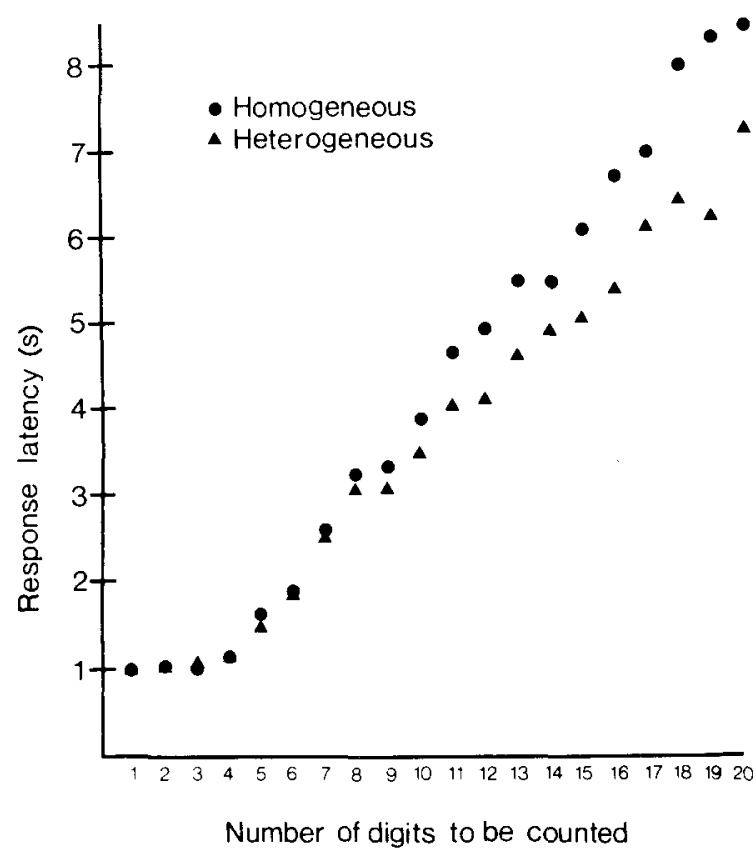

Figure 1. Latency to respond as a function of how many digits were presented.

A trial was defined as being overcounted when too many digits were reported and as being undercounted when too few digits were reported. In the homogeneous condition, $76 \%$ of the miscounted trials were overcounted $(60 / 83)$, and in the heterogeneous condition, $71 \%$ of the miscounted trials were overcounted (32/45). The subjects were classified as overcounters or undercounters on the basis of whether they had overcounted or undercounted more of the trials. In the homogeneous condition, $68 \%$ of the subjects were overcounters $(15 / 22)$, and in the heterogeneous condition, $72 \%$ of the subjects were overcounters $(13 / 18)$. Thus, in both conditions, subjects tended to report too many digits, although there seemed to be individual differences, with one subject, for example, undercounting five trials and not overcounting any.

One aspect of Figure 1 deserves mention. As expected, the curve in Figure 1 increases little, if at all, in the range from 1 to 4 digits. This common finding reflects the span of apprehension (Kaufman, Lord, Reese, \& Volkmann, 1949), in which the magnitude of a small number of objects is perceived directly. However, it seems reasonable to assume that when 5 or more objects are presented, ev-

Table 1

Descriptive Statistics for the Homogeneous and Heterogeneous Conditions of Experiment 1

\begin{tabular}{|c|c|c|c|}
\hline & \multicolumn{2}{|c|}{ Averages } & \multirow{2}{*}{$\begin{array}{c}\text { Number of } \\
\text { Subjects Better } \\
\text { in Heterogeneous } \\
\text { Condition }\end{array}$} \\
\hline & $\overline{\mathrm{HOM}}$ & $\overline{\text { HET }}$ & \\
\hline Response Latency & 4.3 & $3.7^{*}$ & 26/28* \\
\hline Milliseconds per Digit & 463 & $353^{*}$ & $26 / 28 *$ \\
\hline Percent Correct & 85 & $93^{*}$ & $17 / 21^{*}$ \\
\hline
\end{tabular}

Note-Response latency in seconds; HOM = homogeneous condition; HET $=$ heterogeneous condition. $\quad{ }^{*} p<.05$. 
ery object is counted. If this assumption were correct, the intercept of the line through the response latencies in the range from 5 to 20 digits would represent the processing time needed on every trial to, for example, initiate counting and responding. However, as also found in previous experiments (e.g., Aoki, 1977; Chi \& Klahr, 1975), the intercept is negative. Therefore, the original assumption, that when 5 or more digits are presented they are all counted, does not seem to be correct. Figure 1 suggests that when 5 or more objects have to be counted, 4 of these objects are directly apprehended and the rest are counted.

\section{Discussion}

The results suggest that a homogeneous row of digits is more difficult to count than a heterogeneous one. This will be called the "homogeneity" effect. The homogeneity effect seems to be quite robust: It occurred for almost all of the subjects and it increased the response latency by approximately $16 \%$.

One explanation of the homogeneity effect to be dispensed with involves the Stroop effect. When a property of a stimulus differs from that of the correct response, the response is made more slowly and is less accurate (Stroop, 1935). If a Stroop effect were operating on the final spoken response in the homogeneous condition, it would have produced a constant delay unrelated to the number of digits to be counted. Instead, as can be seen in Figure 1, the time to count each individual digit in a homogeneous row increases. Also, a Stroop effect could have occurred as each digit was being counted, whenever that digit differed from the number the subject was supposed to be saying subvocally. Such an effect would increase the time taken to count each digit. However, the probability of a digit's differing from the number that should have been said subvocally was the same in both the homogeneous and heterogeneous conditions. Furthermore, the Stroop effect usually is demonstrated by having the interfering property of the stimulus change from stimulus to stimulus. Thus, there is more reason to expect a Stroop effect in the heterogeneous condition (in which the interfering property changed) than in the homogeneous condition (in which the interfering property did not change). Thus, the Stroop effect does not explain the homogeneity effect.

Another explanation for the homogeneity effect that does not seem to be correct involves the number of digits actually counted. More errors were made in the homogeneous condition, and when subjects made an error they tended to report too many digits. The consequence of these two effects was that more digits were reported in the homogeneous condition. A report of too many digits suggests that some digits were counted more than once. However, the slower responses in the homogeneous condition could not have been a consequence merely of counting more digits. Overall, 5,950 digits were reported in the homogeneous condition and 5,922 digits were reported in the heterogeneous condition. Thus, approximately $0.5 \%$ more digits were reported in the homogeneous condition. This increase is not large enough to explain the $16 \%$ increase in response latency in the homogeneous condition.

Three types of explanations for the homogeneity effect are consistent with the results of Experiment 1. Explanations of the first type will be called fatigue hypotheses. The visual system might fatigue when reading the same object over and over again, leading to slower perception and hence slower counting. Similarly, attention to an object might become more difficult when attending to the same object over and over again.

Explanations of the second type will be called error correction hypotheses. The eye movement to the next object to be counted might not always be accurate. Perhaps when the objects are heterogeneous the eye movement can be easily corrected, whereas when the objects are homogeneous the subject might not know whether the direction of gaze had moved too far or not far enough. The same argument can be made for direction of attention.

Explanations of the third type will be called grouping hypotheses. It seems that the perception of a scene is divided into units preattentively (see Duncan, 1984, for a review). The Gestalt principles of grouping describe the results of this unit formation. One Gestalt principle, called the principle of similarity, is that identical objects tend to be grouped together. By this principle, a homogeneous row of objects would have a stronger tendency to be grouped together than would a heterogeneous row of objects.

There are several reasons why the Gestalt principles of grouping might be involved in counting. People often count objects in small subsets, usually by twos or threes. The subjects in the preceding experiment were instructed to count the digits one by one, but there was no method of verifying that they in fact did so. When the Gestalt principles of grouping act to unify the group of objects, subdividing the group into subsets might be more difficult.

Beckwith and Restle (1966) suggested that, instead of counting objects one by one, subjects select a small subset of the objects and immediately apprehend (or "subitize") the number of objects in that subset. The numbers of objects in the subsets are added together to produce the total. This hypothesis differs from the above hypothesis about counting in subsets. If the subset is formed before it is counted, as Beckwith and Restle suggested, then different subsets presumably could contain differing numbers of objects. Like the other method of counting in groups, this method also requires subdividing the objects.

Finally, one step in counting is to isolate the object to be counted from the other objects. Introspectively, it does not seem to be difficult to isolate the digits. However, isolating individual digits may be more difficult when the Gestalt principles of grouping act to unify the total set of digits to be counted. This third grouping hypothesis can be differentiated from the first two in that the first 
two involve subdividing the objects into smaller subsets, whereas the third involves isolating each object.

\section{EXPERIMENT 2}

The purpose of Experiment 2 was to test the hypotheses generated from Experiment 1 . The homogeneity effect was tested for a variety of configurations of digits. The "normal" configuration (Figure 2a) was a replication of Experiment 1. The "finger" configuration was identical to the normal configuration except that the subject touched the digits being counted with his or her finger. The "spaced" configuration (Figure 2b) was like the normal configuration except that an extra blank space was inserted between each digit. In the "border" configuration (Figure 2c), there was a border of irrelevant digits surrounding the digits to be counted. Finally, in the "3-level" configuration (Figure 2d), the digits were placed on three different levels in the vertical direction.

If the homogeneity effect was a result of fatigue, it should occur in all configurations. However, suppose that the homogeneity effect involved grouping. Presenting the digits on different levels in the 3-level configuration would eliminate grouping by good continuation and allow the digits to be easily subdivided or isolated. If the digits were

444444444444444

(a)

444444444444444

(D)

791395647622 444444444444444

1738828265671

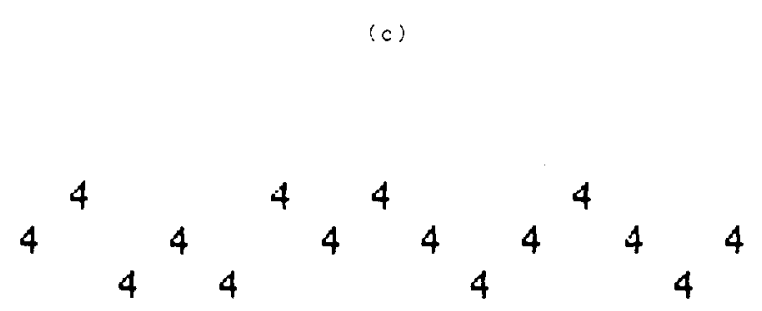

(d)

Figure 2. The configurations tested in Experiment 2: (a) normal and finger, (b) spaced, (c) border, and (d) 3-level. easily subdivided or isolated in both the heterogeneous condition and the homogeneous condition, the homogeneity effect should be eliminated. In the normal, spaced, and border configurations, the stimuli were grouped by good continuation, so the homogeneity effect should occur. In the finger configuration, the stimuli were also grouped together by good continuation, but the subjects were required to point to the digits one by one. Thus, in that configuration, the digits would not be counted in subsets. If the homogeneity effect occurred only as a consequence of attempts to subdivide the digits into subsets, it should not occur in the finger configuration. However, if the homogeneity effect occurred as a consequence of having to isolate each digit, it should occur in the finger configuration.

The homogeneity effect might involve error correction. By varying such factors as the size of the errors and what the subject does when an error occurs, a variety of errorcorrection hypotheses can be constructed. If an error occurred when the subject was trying to move direction of gaze or attention to a 7 , he or she might look for a nearby 7. In the homogeneous condition, there would be a 7 on both sides of the direction of gaze or attention, perhaps creating difficulty. If this hypothesis was correct, there should be a homogeneity effect in all configurations. However, if the errors were small enough and the digits were separated by spaces, the subject might be able to accurately resolve an error in the homogeneous condition by moving to the closest digit. If a separation of digits eliminated the homogeneity effect, there should be no homogeneity effect in the 3-level configuration or the spaced configuration.

Alternatively, the subject might recognize an error in movement by noting that the visual field at the current direction of gaze or attention did not match the intended visual field, and then correct the error by redirecting gaze or attention to the intended visual field. The homogeneous condition would create difficulties because the visual field on one side of a digit would be the same as the visual field on the other side of the digit. Thus, moving the direction of gaze or attention too far would not be distinguishable from moving the direction of gaze or attention not far enough. The relevant visual field could not be of unlimited size: If it were, the edge of the computer screen and the boundaries of the row of digits would be reference points with which one side of a digit could be distinguished from the other in the homogeneous condition. On the other hand, the relevant visual field could not be minutely small: If it was too small, it might contain only a small portion of blank computer screen and provide no evidence concerning the direction of error. If the visual field was large enough, the homogeneity effect might be eliminated in the 3-level configuration, because the visual fields on each side of each digit would be different in that configuration. If the visual field was that large, it would also be large enough to include the border in the border configuration. Thus, the homogeneity effect would also be eliminated in the border configuration. 
Finally, in the finger configuration, the finger is a part of the visual field, albeit a moving part. Therefore, if the homogeneity effect involved error correction, it might not occur in the finger configuration.

\section{Method}

Experiment 2 differed from Experiment 1 in the following details. Design. There were five groups of subjects, corresponding to the five different configurations of digit presentation, with 12 subjects in each group. Altogether, 60 subjects were tested.

Stimuli. The five configurations are illustrated in Figure 2. In the spaced configuration, the space between digits doubled the total length of the presentation of the normal configuration. In the border configuration, the digits on the border were selected randomly and spaced irregularly to prevent subjects from counting the border and inferring the number of digits. The 3-level configuration was like the spaced configuration, except that there were three possible levels on which the digits could be placed. The level on which a digit was placed was determined randomly with the restriction that two successive digits not appear on the same level and the first digit be presented on the middle level. In all of the configurations, the first digit was presented at the far left of the computer screen.

One of the configurations required more time between trials for the computer to construct the stimuli than was required in Experiment 1 . The length of time between trials was held constant across configurations by inserting delays between trials for the other configurations. The number of digits presented on a trial varied from 6 to 20 . Because the homogeneity effect did not seem to exist when fewer than 6 digits had to be counted, the trials with 5 or fewer digits would not have been informative in revealing the existence or nonexistence of the homogeneity effect.

\section{Results}

Descriptive statistics are presented in Table 2. Consider first the overall response latency. An ANOVA was per-

Table 2

Descriptive Statistics for the Homogeneous and Heterogeneous Conditions of Experiment 2

\begin{tabular}{|c|c|c|c|}
\hline & \multicolumn{2}{|c|}{ Averages } & \multirow{2}{*}{$\begin{array}{c}\text { Number of } \\
\text { Subjects Better } \\
\text { in Heterogeneous } \\
\text { Condition } \\
\end{array}$} \\
\hline & HOM & HET & \\
\hline \multicolumn{4}{|c|}{ Response Latency (in Seconds) } \\
\hline Normal & 5.0 & $4.2 *$ & $12 / 12 *$ \\
\hline Finger & 4.7 & $4.1^{*}$ & $11 / 12^{*}$ \\
\hline Spaced & 4.4 & $3.8^{*}$ & $11 / 12 *$ \\
\hline Border & 6.1 & $5.1^{*}$ & $11 / 12^{*}$ \\
\hline 3 Level & 3.2 & 3.2 & $6 / 12$ \\
\hline \multicolumn{4}{|c|}{ Milliseconds per Digit } \\
\hline Normal & 404 & $333^{*}$ & $10 / 12^{*}$ \\
\hline Finger & 345 & 315 & $10 / 12^{*}$ \\
\hline Spaced & 344 & 297* & $10 / 12^{*}$ \\
\hline Border & 428 & 386 & $10 / 12 *$ \\
\hline 3 Level & 221 & 228 & $7 / 12$ \\
\hline \multicolumn{4}{|c|}{ Percent Correct } \\
\hline Normal & 81 & 84 & $5 / 9$ \\
\hline Finger & 90 & 92 & $4 / 10$ \\
\hline Spaced & 84 & 92 & $7 / 9$ \\
\hline Border & 83 & 94* & $9 / 10^{*}$ \\
\hline 3 Level & 88 & 87 & $5 / 10$ \\
\hline
\end{tabular}

Note-HOM = homogeneous condition; HET = heterogeneous condition. $\quad{ }^{*} p<.05$. formed comparing all five configurations and using the difference between the homogeneous condition and the heterogeneous condition as the dependent measure. There was an overall effect of configuration $[F(4,55)=6.47]$, suggesting that the configurations should be analyzed separately. There was a homogeneity effect in the normal configuration $[t(11)=4.75]$, the finger configuration $[t(11)=3.47]$, the spaced configuration $[t(11)=$ 6.77], and the border configuration [ $t(11)=4.59$ ] (all $p s<.01$ ). When only these four configurations were considered in an ANOVA, there was no overall effect of configuration $[F(3,44)=1.31]$. Thus, there was no detectable difference in the size of the homogeneity effect for these four configurations. In the 3-level configuration, however, there was no indication of a homogeneity effect $[t(11)=.05]$.

The dependent measure of estimated time to count each digit was not as sensitive as overall response latency. There was a homogeneity effect for this measure in the normal configuration $[t(11)=2.89, p<.02]$ and in the spaced configuration $[t(11)=3.01, p<.02]$. The finger configuration and the border configuration each had 1 subject for whom the estimated time to count each digit was much longer in the heterogeneous condition than in the homogeneous condition (despite a slower overall response latency in the homogeneous condition). Because of these 2 subjects, the difference between the homogeneous condition and the heterogeneous condition was not statistically significant in those configurations. However, the proportion of subjects showing the homogeneity effect in those two configurations was statistically significant $(10 / 12, p<.05)$. Again, there was no sign of a homogeneity effect in the 3-level configuration.

Finally, consider the dependent measure of accuracy. The difference between the homogeneous condition and the heterogeneous condition was statistically significant in the border configuration $[t(11)=3.88, p<.01]$, providing additional evidence for a homogeneity effect in that configuration. For the 3-level configuration, the difference between the homogeneous condition and the heterogeneous condition was not statistically significant $[t(11)=.30]$, which is consistent with the lack of a homogeneity effect found in that configuration. For the remaining three configurations, the subjects were more accurate in the heterogeneous condition than in the homogeneous condition, but the difference was not statistically significant. The important implication of the latter result is that the faster responses in the heterogeneous conditions were not merely a consequence of a speed-accuracy tradeoff.

There again was no detectable difference in overcounting between the homogeneous and heterogeneous conditions, so the data from these two conditions were combined. For the four configurations excluding the 3-level configuration, the subjects again tended to overcount the number of digits: The number of digits was overcounted on $80 \%$ of the miscounted trials (144/179), and $84 \%$ of the subjects were overcounters $(31 / 37)$. In the 3-level con- 
figuration, the picture is not as clear. Six subjects were overcounters and only 3 were undercounters (67\%). However, the 3 undercounters made most of the errors, so only $37 \%$ of the miscounted trials (17/46) were overcounted.

\section{Discussion}

There was no indication that the homogeneity effect was reduced by spacing the digits further apart on the computer screen, by placing a heterogeneous border around the digits, or by having the subjects point to each digit with a finger. However, the homogeneity effect was greatly reduced if not eliminated by presentation of the digits on three different levels.

The hypothesis that perception or attention fatigues when the same digit is perceived over and over again predicted that the homogeneity effect should have occurred in all of the configurations. The lack of an effect in the 3-level configuration suggests that the homogeneity effect is not a consequence of fatigue.

Hypotheses involving error correction have difficulty explaining both the elimination of the homogeneity effect in the 3-level configuration and the presence of the homogeneity effect in the other configurations. Errors in movement of the direction of gaze or attention might be resolved by moving to the closest object when the objects are separated. This hypothesis explains the lack of a homogeneity effect in the 3-level configuration, but it predicts that the homogeneity effect should have been eliminated in the spaced configuration as well. Another possibility is that errors might be correctable when the visual field on one side of a digit is different from that on the other side of the digit. This hypothesis explains the lack of a homogeneity effect in the 3-level configuration, but it predicts that the homogeneity effect should have been eliminated in the border configuration as well.

Thus, several hypotheses involving error correction were considered, and none were consistent with the results of this experiment. These hypotheses were not exhaustive, which is to say that other hypotheses involving error correction could exist and one of these unimagined hypotheses might be correct. All that can be said is that the spaced, border, and finger configurations constituted a variety of unsuccessful attempts to eliminate the homogeneity effect on the basis of the assumption that there was a problem in error correction. Only the 3-level configuration eliminated the homogeneity effect, and the elimination in that configuration can be explained by grouping hypotheses.

The results of Experiment 2 suggest that the Gestalt principles of grouping are involved in the homogeneity effect. In four of the configurations (normal, finger, spaced, and border), the digits were grouped together by the Gestalt principle of good continuation. In those configurations there was a homogeneity effect. In the 3-level configuration, the digits were not grouped together by the Gestalt principle of good continuation. In that configuration there was no homogeneity effect. Apparently, the digits in the 3-level configuration were easily subdivided or isolated in both the homogeneous and heterogeneous conditions.

Two of the grouping hypotheses were based upon the notion that the subjects might be subdividing the digits into subsets. In the finger configuration, subjects had to point to the digits one by one, so presumably they were not subdividing the digits. Nonetheless, a homogeneity effect still occurred. Apparently, isolating each digit of a homogeneous row is difficult.

Finally, response latencies for both the homogeneous and heterogeneous conditions were faster in the 3-level configuration than in the other configurations. The 3-level configuration could have been faster than the other configurations in the homogeneous condition because of the lack of a homogeneity effect in that configuration. Therefore, it is appropriate to compare just the heterogeneous conditions. In an overall ANOVA of response latencies in the heterogeneous condition, the five configurations were different $[F(4,55)=3.40, p<.05]$, and in pairwise comparisons, the 3-level configuration was counted more quickly than all of the other configurations [t(22) $>2.45, p<.05]$. This result suggests that (1) even a heterogeneous row of digits is difficult to isolate or subdivide, and (2) isolating or subdividing these digits can be improved by presenting the digits in a way that avoids grouping by good continuation. However, confidence in this result should be moderated by the fact that accuracy tended to be lower in the 3-level configuration than in the other configurations, suggesting the possible presence of a speed-accuracy tradeoff.

\section{EXPERIMENT 3}

There were several reasons for wanting to retest the 3-level configuration. First, the left-to-right order of report was obvious to the experimenters and was suggested by the dash that signaled where to start counting. However, the subjects could have counted the digits in the 3-level configuration level by level rather than from left to right. Because (1) there would rarely be more than 6 digits in a level, and (2) there seems to be little or no homogeneity effect for rows of 6 or fewer digits, such a strategy might have eliminated the homogeneity effect. ${ }^{1}$ To prevent the use of this strategy in Experiment 3, subjects were required to point to the digits one by one, in order to verify that the digits were being counted from left to right. Second, the post hoc finding in Experiment 2 that subjects could count more quickly overall with the 3-level configuration provides additional support for a role of Gestalt principles of grouping in counting. To confirm this finding, performance on the 3-level configuration was compared with that on the spaced configuration. To facilitate this comparison, a completely within-subject design was used.

The most sensitive measure in the previous experiment was overall response latency. An examination of Figure 1 reveals that the difference between the homogeneous con- 
dition and the heterogeneous condition was small when fewer than approximately 9 digits had to be counted. In Experiment 2, the difference was again small when fewer than 9 digits had to be counted, with the average being 156,456 , and $244 \mathrm{msec}$, respectively, when 6,7 , and 8 digits had to be counted (collapsing across the four configurations that showed the homogeneity effect). To accommodate the testing of both configurations without greatly increasing the total time needed, only trials with 9 to 20 digits were presented.

\section{Method}

Experiment 3 differed from Experiment 2 in the following details. Subjects. Twelve subjects participated.

Design. The 3-level configuration and the spaced configuration were tested. The spaced configuration was chosen for testing because, of the configurations tested in Experiment 2, the spaced configuration was most like the 3-level configuration. Each subject was tested for both of these configurations in both the homogeneous and the heterogeneous conditions. In addition, the subjects always pointed to each digit as in the finger configuration of Experiment 2.

Stimuli. To prevent the subject's hand from blocking the initial view of the digits, the first digit in the 3-level configuration appeared on the bottom level. There were 12 trials per condition, each containing 9 to 20 digits, for a total of 48 experimental trials. In addition, there were 6 practice trials. There were no long pauses between trials as there had been in Experiment 2.

\section{Results}

The descriptive statistics are presented in Table 3. The only discriminating dependent measure was overall response latency. A $2 \times 2$ ANOVA was performed testing the spaced configuration versus the 3-level configuration and the homogeneous condition versus the heterogeneous condition. There was an interaction $[F(1,11)=$ $12.13, p<.01]$, suggesting that the two configurations should be analyzed separately. The spaced configuration produced a homogeneity effect $[t(11)=4.39, p<.01]$, whereas the 3-level configuration did not $[t(11)=.82]$.

Table 3

Descriptive Statistics for the Homogeneous and Heterogeneous Conditions of Experiment 3

\begin{tabular}{|c|c|c|c|}
\hline & \multicolumn{2}{|c|}{ Averages } & \multirow{2}{*}{$\begin{array}{c}\text { Number of } \\
\text { Subjects Better } \\
\text { in Heterogeneous } \\
\text { Condition }\end{array}$} \\
\hline & HOM & HET & \\
\hline & \multicolumn{3}{|c|}{ Response Latency (in Seconds) } \\
\hline $\begin{array}{l}\text { Spaced } \\
3 \text { Level }\end{array}$ & $\begin{array}{l}4.5 \\
3.6\end{array}$ & $\begin{array}{l}4.2^{*} \\
3.6\end{array}$ & $\begin{array}{c}11 / 12^{*} \\
9 / 12\end{array}$ \\
\hline \multicolumn{4}{|c|}{ Milliseconds per Digit } \\
\hline $\begin{array}{l}\text { Spaced } \\
3 \text { Level }\end{array}$ & $\begin{array}{l}308 \\
242\end{array}$ & $\begin{array}{l}296 \\
229\end{array}$ & $\begin{array}{l}7 / 12 \\
7 / 12\end{array}$ \\
\hline \multicolumn{4}{|c|}{ Percent Correct } \\
\hline $\begin{array}{l}\text { Spaced } \\
3 \text { Level } \\
\end{array}$ & $\begin{array}{l}92 \\
95 \\
\end{array}$ & $\begin{array}{l}93 \\
94 \\
\end{array}$ & $\begin{array}{l}2 / 7 \\
1 / 4 \\
\end{array}$ \\
\hline
\end{tabular}

Note-HOM = homogeneous condition; HET = heterogeneous condition. $\quad * p<.05$.
The dependent measure of estimated time to count each digit did not always detect the homogeneity effect in Experiment 2 . The reliability of this measure was further impaired in Experiment 3 by the exclusion of trials of 6 to 8 digits, which reduced both the number of trials and the range upon which the estimate was based. Therefore, this dependent measure was not necessarily appropriate for this experiment, and the lack of statistical significance in the spaced configuration $[t(11)=.92]$ and the 3-level configuration $[t(11)=1.39]$ should not be interpreted as evidence for or against the existence of the homogeneity effect. As in Experiment 2, the measure of accuracy also did not differentiate the homogeneous and heterogeneous conditions. The important result concerning the latter measure is that the homogeneity effect found for response latency in the spaced configuration was not a consequence of a speed-accuracy tradeoff.

The same pattern of counting errors appeared as in the previous experiments. In the spaced configuration, $81 \%$ of the miscounted trials (17/21) were overcounted and $71 \%$ of the subjects $(5 / 7)$ were overcounters. In the $3-$ level configuration, $60 \%$ of the subjects $(3 / 5)$ were overcounters. However, because of one prolific undercounter, only $44 \%$ of the miscounted trials $(7 / 16)$ were overcounted. There was no observable difference between the heterogeneous and homogeneous conditions in either configuration.

The final comparison between the spaced configuration and the 3-level configuration considers only the heterogeneous condition. For overall response latency, the difference between these two configurations was highly significant $[t(11)=12.04, p<.001]$, with every subject responding faster in the 3 -level configuration. These two configurations also differed for the dependent measure of estimated time to count each digit $[t(11)=7.52$, $p<.001]$. Accuracy was also slightly higher in the 3level configuration than in the spaced configuration. This difference was not statistically significant, but it indicates that the findings concerning response latency were not the result of a speed-accuracy tradeoff.

\section{Discussion}

The results of this experiment replicated and extended several findings from Experiment 2. First, the homogeneity effect was present when the digits were spaced and when subjects pointed to the digits. Second, the homogeneity effect was eliminated in the 3-level configuration. This elimination was not a result of subjects' counting the digits level by level instead of from left to right. Finally, the 3-level configuration facilitated counting even in the heterogeneous condition. This facilitation was not the result of a speed-accuracy tradeoff. Furthermore, it occurred even though the digits were counted one by one. These effects are consistent with the hypothesis that the Gestalt principles of grouping impair the process of isolating each digit. 


\section{GENERAL DISCUSSION}

\section{Empirical Findings}

There were three empirical findings of importance. First, homogeneous rows of digits were more difficult to count than heterogeneous rows. This homogeneity effect was robust, occurring as it did for almost all subjects and across a variety of situations. Second, the homogeneity effect did not occur when the digits were presented on three different levels. Third, digits evenly spaced on one row were more difficult to count than digits presented on three different levels, even in the heterogeneous condition.

\section{Explanations of the Homogeneity Effect}

Several explanations of the homogeneity effect were not supported by the results. To review these, the Stroop effect did not explain why performance would be better in the heterogeneous condition than in the homogeneous condition. Explanations involving fatigue in repetitively perceiving or attending to identical digits did not explain why the homogeneity effect was eliminated in the 3-level configuration. Two hypotheses involving error correction could explain the lack of a homogeneity effect in the 3level configuration. One hypothesis was that when the digits were separated, the subject could correctly resolve errors by moving to the closest digit. This hypothesis incorrectly predicted that there would not be a homogeneity effect in the spaced configuration. The second hypothesis was that differing visual fields on each side of a digit allowed subjects to distinguish moving too far from not moving far enough. This hypothesis incorrectly predicted that there would not be a homogeneity effect in the border configuration.

In these experiments, counting was impaired to the extent that the digits were unified by Gestalt principles of good continuation and similarity. Counting sometimes involves subdividing the digits into subsets, and the process of subdividing digits might be impaired when the digits are grouped together. However, even when digits were counted one by one, counting was impaired when the digits were grouped together. Thus, the hypothesis that best accounted for the results of these experiments was that the digits were not easily isolated when they were unified by Gestalt principles of grouping.

\section{The Effect of Good Continuation}

Digits on three levels were more quickly counted than digits in a single row. This finding was unexpected. A completely unorganized set of digits is difficult to count because the digits are difficult to order. One might thereby conclude that the more organized a set of digits is, the easier that set of digits would be to count. However, the ultimate in organization would be to align the digits in one evenly spaced row. This "most organized" configuration is also difficult to count, apparently because it elicits a unified grouping of the digits according to Gestalt principles. A set of digits seems to be most easily counted when it is moderately disorganized: There must be enough organization to allow ordering of the digits and enough disorganization to prevent the Gestalt principles of grouping from operating.

\section{Shifting Attention in Counting}

When the number of objects to be counted exceeds the span of apprehension, a person apparently cannot attend to all of the objects at once and simultaneously count them. Instead, my subjective impression is that when I count objects one by one I attend to the objects one by one. Assuming that a person is attending to one object (or a subset of objects), how does the person know the location of the next object (or subset of objects) to which attention should be shifted? One hypothesis would be that attention glides smoothly along the row of digits and the digits are counted as they are perceived. This explanation has several difficulties. First, it does not match the introspection of attending to each digit one at a time. Second, this hypothesis provides no explanation of the homogeneity effect. Third, this hypothesis seems to predict slower counting in the 3-level configuration, in which the location of the next digit is not as predictable as it is in the other configurations. Finally, people who are convinced by the evidence for an "object-based" theory of attention (e.g., Duncan, 1984) would argue that this explanation is not object-based.

Suppose, then, that attention shifts from object to object. If attention is upon one object, how does the subject know the location of the next object, so that attention can be shifted to that object? If unattended stimuli were not processed, the subject would not know the location of the next object. Furthermore, the subject could not discover the location of the next object without first attending to that object. In fact, unattended stimuli are processed (Cherry, 1953; Eich, 1984; Treisman, 1964). One purpose of processing unattended stimuli presumably is to guide the shifting of attention to unattended stimuli. This guidance is most obvious when attention is involuntarily shifted to an unattended object, but this guidance would also be useful for voluntary shifts of attention such as those that occur when scanning a scene. If attention is directed toward objects rather than toward points in space, these objects must be defined in this preattentive representation. The Gestalt principles of grouping describe the outcome of the processes used to form objects.

Thus, it seems quite reasonable to suppose that there is a preattentive representation of the environment that is used to guide the shifting of attention. When the subject is attending to one digit in a row of digits, the location of the next digit is contained in this preattentive representation. However, before attention can be shifted to the next digit, the digit must be isolated and recognized as an individual object in this preattentive representation. When the Gestalt principles group the digits together, the process of recognizing individual digits as objects is more difficult. 
The explanation of the homogeneity effect derived from these experiments was unusual in that it concerned the Gestalt principles of grouping but did not match introspective experience. The Gestalt principles were first discovered and demonstrated by using introspection, whereas in the present experiments the effect of the Gestalt principles was inferred from subjects' performance. Introspectively, the digits did not seem difficult to isolate. However, the inferred conclusion was that the process of isolating the digits was impaired. The above model can account for the discrepancy between introspection and this conclusion. According to the above model, the difficulty in isolating a digit occurred preattentively. Therefore, by the time a digit was available for attention, it had already been isolated. Thus, the preattentive difficulty in isolating the digit was not introspectively observable.

\section{Summary}

Counting is impaired when objects in a row are identical. This was called the homogeneity effect. Counting is also impaired when the objects to be counted are evenly spaced and aligned in one row. A hypothesis consistent with the findings was that the Gestalt principles of grouping act against the process of isolating each object to be counted. A model was proposed in which (1) a preattentive representation of the environment guides the shifting of attention, and (2) the grouping by Gestalt principles impairs the process of isolating objects in this preattentive representation.

\section{REFERENCES}

Aokı, T. (1977). On the counting process of patterned dots. Tohoku Psychologica Folia, 36, 15-22.

Beckwith, M., \& Restle, F. (1966). Process of enumeration. Psychological Review, 73, 437-444.

Cherry, E. C. (1953). Some experiments on the recognition of speech, with one and two ears. Journal of the Acoustical Society of America, 25, 975-979.

CHI, M. T. H., KLAHR, D. (1975). Span and rate of apprehension in children and adults. Journal of Experimental Child Psychology, $19,434-439$.

DUNCAN, J. (1984). Selective attention and the organization of visual information. Journal of Experimental Psychology: General, 113, 501-517.

EICH, E. (1984). Memory for unattended events: Remembering with and without awareness. Memory \& Cognition, 12, 105-111.

Kaufman, E. L., Lord, M. W., Reese, T. W., \& Volkmann, J. (1949). The discrimination of visual number. American Journal of Psychology, 62, 498-525.

PoTter, M. C., \& LEVY, E. I. (1968). Spatial enumeration without counting. Child Development, 39, 265-272.

STROOP, J. R. (1935). Studies of interference in serial verbal reactions. Journal of Experimental Psychology, 18, 643-662.

Treisman, A. M. (1964). Monitoring and storage of irrelevant messages in selective attention. Journal of Verbal Learning \& Verbal Behavior, 3, 449-459.

\section{NOTE}

1. I thank Alfred Kristofferson for noting this possibility.

(Manuscript received May 27, 1986;

revision accepted for publication September 25, 1986.) 